\title{
Selected papers on medicinal chemistry
}

\author{
Vittorio Pace ${ }^{1}$ (D) Michael Schnürch ${ }^{2}$
}

Received: 18 April 2018/ Accepted: 9 May 2018/Published online: 15 May 2018

(C) Springer-Verlag GmbH Austria, part of Springer Nature 2018

The 4th Edition of the EFMC Young Medicinal Chemist Symposium (EFMC-YMCS) organized by the European Federation for Medicinal Chemistry took place in Vienna (Austria) between August 31st and September 1st 2018. The event has been organized in connection to the 7 th Edition of the EFMC International Symposium on Advances in Synthetic and Medicinal Chemistry.

Prof. Thierry Langer (University of Vienna) chaired this attractive 2-day event with the advice of Prof. Koen Augustyns (EFMC President) and Prof. Michael Schnürch (TU Wien). The meeting benefited from the presence of distinguished medicinal chemists from both academia and industries. Over 170 scientists coming from 30 countries attended the symposium constituted by two key note lectures, 14 oral communications by invited prize winners from national young medicinal chemist meetings in Europe, four additional selected oral communications from submitted abstracts, 20 flash poster presentations and more than 100 posters.

Three awards were assigned to the best presentation (Daniel Merk-ETH Zürich, Switzerland-sponsored by EFMC and Actelion), best poster (Matthieu Place-ICOA CNRS, France-sponsored by Molecules and RSC), and Public's Choice Prize (Vittorio Pace-University of Vienna, Austria, sponsored by Roche-F. Hoffman).
The topics covered almost all the aspects of modern medicinal chemistry encompassing drug design, synthesis, and biological evaluation in major therapeutic areas, such as oncology, antiinfective drugs, inflammation, and metabolic disorders.

Overall, the meeting aimed at highlighting the actual panorama of young investigators in the broader context of medicinal chemistry, representing a unique opportunity to foresee perspectives on future developments of our chemistries and, enabling to straighten contacts with colleagues operating in analogous fields.

In this dedicated issue of the journal a selection of eight papers (reviews, communications and full papers) from invited authors who attended the symposium with the intention of further disseminating the science presented at the EFMC-YMCS meeting are presented.

We take the opportunity to manifest our gratitude to the Editor-in-Chief of the journal, Prof. Dr. Peter Gärtner and the Managing Editor, Dr. Christian Hametner for their kind advice and support for publishing this issue. As the Guest Editors, we thank all the reviewers for the constructive comments that improved the quality of the manuscripts and Springer Vienna for the contribution to the publication of this special issue.

Vittorio Pace

vittorio.pace@univie.ac.at

$\triangle$ Michael Schnürch

michael.schnuerch@tuwien.ac.at

1 Department of Pharmaceutical Chemistry, University of Vienna, Vienna, Austria

2 Institute of Applied Synthetic Chemistry, TU Wien, Vienna, Austria 